\title{
Correction to: Multi-relay selection in energy-harvesting cooperative wireless networks: game-theoretic modeling and analysis
}

\author{
Mohammed W. Baidas ${ }^{1}$ (D) Emad Alsusa ${ }^{2} \cdot$ Motasem Alfarra $^{3} \cdot$ Mubarak Al-Mubarak $^{4}$
}

Published online: 25 November 2019

(c) Springer Science+Business Media, LLC, part of Springer Nature 2019

\section{Correction to:}

\section{Telecommunication Systems}

\section{https://doi.org/10.1007/s11235-019-00611-6}

Unfortunately, the original publication contains production errors. We would like to correct the errors as given below:

(a) The fourth author email address should read as "almubarak.13@osu.edu” instead of “Almubarak.13@ osu.edu.kw."

(b) The following equations $5,14,17,19,25,26,27,32,36$, $40,42,46,48,49$ should read as below.

(c) The equations in the algorithm 2,3 should read as below.

The original article has been updated.

$$
\begin{aligned}
p_{\xi_{r_{k}}^{\zeta}}(\xi) & \triangleq \mathbb{P}\left[\xi_{r_{k}}^{\zeta}=\xi\right] \\
& =\sum_{m=0}^{\infty} \mathbb{P}\left[\xi_{r_{k}}^{\zeta}=\xi \mid m\right] \cdot \mathbb{P}\left[\mathcal{N}_{r_{k}}^{\zeta}=m\right],
\end{aligned}
$$

The original article can be found online at https://doi.org/10.1007/ s11235-019-00611-6.

Mohammed W. Baidas

m.baidas@ku.edu.kw

Emad Alsusa

e.alsusa@manchester.ac.uk

Motasem Alfarra

motasem.alfarra@kaust.edu.sa

Mubarak Al-Mubarak

almubarak.13@osu.edu

1 Department of Electrical Engineering, College of Engineering and Petroleum, Kuwait University, Kuwait City, Kuwait

2 School of Electrical and Electronic Engineering, University of Manchester, Manchester, UK

3 Department of Electrical Engineering, King Abdullah University of Science and Technology, Thuwal, Saudi Arabia

4 Department of Electrical and Computer Engineering, Ohio State University, Columbus, OH, US

$$
\begin{aligned}
& \mathcal{R}_{i}^{\zeta}\left(\mathbf{E}_{C_{i}}^{\zeta}, \mathcal{I}_{i}^{\zeta}\right)=\frac{1}{N+1} \log _{2}\left(1+\frac{E_{B_{i}}^{\zeta}\left|h_{i, d}^{\zeta}\right|^{2}}{N_{0}}\right. \\
& \left.+\sum_{k=1}^{K} \mathcal{I}_{i, k}^{\zeta} \frac{E_{B_{i}}^{\zeta} E_{C_{i, k}}^{\zeta}\left|h_{i, k}^{\zeta}\right|^{2}\left|h_{k, d}^{\zeta}\right|^{2}}{N_{0} \varrho_{N}\left(E_{B_{i}}^{\zeta}\left|h_{i, k}^{\zeta}\right|^{2}+E_{C_{i, k}}^{\zeta}\left|h_{k, d}^{\zeta}\right|^{2}+N_{0}\right)}\right),
\end{aligned}
$$

$$
\begin{aligned}
& \mathcal{R}_{i}\left(\mathbf{E}_{R}^{\zeta}, \mathbf{n}_{R}^{\zeta}\right)=\frac{1}{N+1} \log _{2}\left(1+\frac{E_{B_{i}}^{\zeta}\left|h_{i, d}^{\zeta}\right|^{2}}{N_{0}}\right. \\
& \left.+\sum_{k=1}^{K} \mathcal{I}_{i, k}^{\zeta} \frac{E_{B_{i}}^{\zeta}\left(\frac{E_{r_{k}}^{\zeta}}{n_{r_{k}}^{\zeta}}\right)\left|h_{i, k}^{\zeta}\right|^{2}\left|h_{k, d}^{\zeta}\right|^{2}}{N_{0 \varrho N}\left(E_{B_{i}}^{\zeta}\left|h_{i, k}^{\zeta}\right|^{2}+\left(\frac{E_{r_{k}}^{\zeta}}{n_{r_{k}}^{\zeta}}\right)\left|h_{k, d}^{\zeta}\right|^{2}+N_{0}\right)}\right),
\end{aligned}
$$

$$
\Delta \mathcal{R}_{i, k}\left(E_{r_{k}}^{\zeta}, n_{r_{k}}^{\zeta}\right)=\frac{1}{N+1} \log _{2}\left(1+\frac{\left(\frac{E_{r_{k}}^{\zeta}}{n_{r_{k}}^{\zeta}}\right) \cdot \Omega_{i, k}^{\zeta}}{\left(\frac{E_{r_{k}}^{\zeta}}{n_{r_{k}}^{\zeta}}\right)+\Upsilon_{i, k}^{\zeta}}\right),
$$

$$
\beta_{i, k}^{\zeta}\left(\theta_{k}\right) \frac{p_{\mathcal{E}_{r_{k}}^{\zeta}}\left(\phi_{i, k}^{\zeta} \mid \theta_{r_{k}}\right) p_{r_{k}}^{\zeta-1}\left(\theta_{r_{k}}\right)}{\sum_{\theta_{r_{k}} \in \Theta} p_{\mathcal{E}_{r_{k}}^{\zeta}}\left(\phi_{i, k}^{\zeta} \mid \theta_{r_{k}}\right) p_{r_{k}}^{\zeta-1}\left(\theta_{r_{k}}\right)}, \forall \theta_{r_{k}} \in \Theta
$$


$p_{r_{k}}^{\zeta}\left(\theta_{r_{k}}\right)=\frac{1}{N} \sum_{i=1}^{N}\left[\mathcal{I}_{i, k}^{\zeta} \beta_{i, k}^{\zeta}\left(\theta_{r_{k}}\right)+\left(1-\mathcal{I}_{i, k}^{\zeta}\right) p_{r_{k}}^{\zeta-1}\left(\theta_{r_{k}}\right)\right]$,

$p_{r_{k}}^{\zeta}\left(\theta_{r_{k}}\right)=p_{r_{k}}^{\zeta-1}\left(\theta_{r_{k}}\right)+\frac{1}{N} \sum_{i=1}^{N} \mathcal{I}_{i, k}^{\zeta}\left(\frac{p_{\mathcal{E}_{r_{k}}^{\zeta}}\left(\phi_{i, k}^{\zeta} \mid \theta_{r_{k}}\right)}{\Lambda\left(\phi_{i, k}^{\zeta}\right)}\right) p_{r_{k}}^{\zeta-1}\left(\theta_{r_{k}}\right)$,

$\forall \theta_{r_{k}} \in \Theta$ and $\forall k \in\{1,2, \ldots, K\}$,

$$
\begin{aligned}
& \mathbb{U}_{i, k}\left(\mathbf{p}_{r_{k}}^{\zeta}, n_{-i, k}^{\zeta}\right) \\
& =\sum_{\theta_{r_{k}} \in \Theta} \sum_{\xi=0}^{\infty} \mathcal{U}_{i, k}\left(\xi, n_{-i, k}^{\zeta}+\mathcal{I}_{i, k}^{\zeta}\right) p_{\xi_{r_{k}}^{\zeta}}\left(\xi \mid \theta_{r_{k}}\right) p_{r_{k}}^{\zeta}\left(\theta_{r_{k}}\right) \\
& \left.\mathbb{U}_{i, k}\right|_{\mathcal{I}_{i}^{\zeta}=\omega_{q}} \\
& =\omega_{q, k} \sum_{\theta_{r_{k}} \in \Theta} \sum_{\xi=0}^{\infty} \mathcal{U}_{i, k}\left(\xi, n_{s_{i}, r_{k}}^{\zeta}+\left.\mathcal{J}_{i, k}^{\zeta}\right|_{\mathcal{I}_{i}^{\zeta}=\omega_{q}}+\omega_{q, k}\right) p_{\xi_{\zeta_{k}}^{\zeta}}\left(\xi \mid \theta_{r_{k}}\right) p_{r_{k}}^{\zeta}\left(\theta_{r_{k}}\right) .
\end{aligned}
$$

$\mathcal{I}_{i}^{\zeta, *}\left(\mathbf{p}_{R}^{\zeta}, \mathbf{n}_{-i}^{\zeta}\right)=\sum_{k=1}^{K} \underset{\mathcal{I}_{i, k}^{\zeta} \in\{0,1\}}{\operatorname{argmax}} \mathcal{I}_{i, k}^{\zeta} \cdot \mathbb{U}_{i, k}\left(\mathbf{p}_{r_{k}}^{\zeta}, n_{-i, k}^{\zeta}\right)$

$$
\begin{aligned}
& \mathbb{U}_{i, k}\left(\mathbf{p}_{r_{k}}^{\zeta}, n_{s_{i}, r_{k}}^{\zeta}\right) \\
& =\sum_{\theta_{r_{k}} \in \Theta} \sum_{\xi=0}^{\infty} \mathcal{U}_{i, k}\left(\xi, n_{s_{i}, r_{k}}^{\zeta}+\left.\mathcal{J}_{i, k}^{\zeta}\right|_{\mathcal{I}_{i, k}^{\zeta}=1}+1\right) p_{\xi_{r_{k}}^{\zeta}}\left(\xi \mid \theta_{r_{k}}\right) p_{r_{k}}^{\zeta}\left(\theta_{r_{k}}\right) .
\end{aligned}
$$

$$
\begin{aligned}
& \Delta \mathcal{R}_{i}\left(\mathbf{E}_{R}^{\zeta}, \mathbf{n}_{R}^{\zeta}\right)=\frac{1}{N+1}\left[\operatorname { l o g } _ { 2 } \left(1+\frac{E_{B_{i}}^{\zeta}\left|h_{i, d}^{\zeta}\right|^{2}}{N_{0}}\right.\right. \\
& \left.+\sum_{k=1}^{K} \mathcal{I}_{i, k}^{\zeta} \frac{E_{B_{i}}^{\zeta}\left(\frac{E_{r_{k}}^{\zeta}}{n_{r_{k}}^{\zeta}}\right)\left|h_{i, k}^{\zeta}\right|^{2}\left|h_{k, d}^{\zeta}\right|^{2}}{N_{0} \varrho_{N}\left(E_{B_{i}}^{\zeta}\left|h_{i, k}^{\zeta}\right|^{2}+\left(\frac{E_{r_{k}}^{\zeta}}{n_{r_{k}}^{\zeta}}\right)\left|h_{k, d}^{\zeta}\right|^{2}+N_{0}\right)}\right) \\
& \left.-\log _{2}\left(1+\frac{E_{B_{i}}^{\zeta}\left|h_{i, d}^{\zeta}\right|^{2}}{N_{0}}\right)\right] .
\end{aligned}
$$

$\mathbb{U}_{i, k}\left(\mathbf{p}_{r_{k}}^{\zeta}\right)=\sum_{\theta_{r_{k}} \in \Theta} \sum_{\xi=0}^{\infty} \mathcal{U}_{i, k}\left(\xi, \mathcal{I}_{i, k}^{\zeta}\right) p_{\xi_{r_{k}}^{\zeta}}\left(\xi \mid \theta_{r_{k}}\right) p_{r_{k}}^{\zeta}\left(\theta_{r_{k}}\right)$.

$$
\begin{aligned}
& \mathbb{U}_{i, k}\left(n_{s_{i}, r_{k}}^{\zeta}\right) \\
& =\sum_{\theta_{r_{k}} \in \Theta} \sum_{\xi=0}^{\infty} \mathcal{U}_{i, k}\left(\xi, n_{s_{i}, r_{k}}^{\zeta}+\mathcal{I}_{i, k}^{\zeta}\right) p_{\xi_{\zeta_{k}}}\left(\xi \mid \theta_{r_{k}}\right) p_{r_{k}}^{0}\left(\theta_{r_{k}}\right),
\end{aligned}
$$

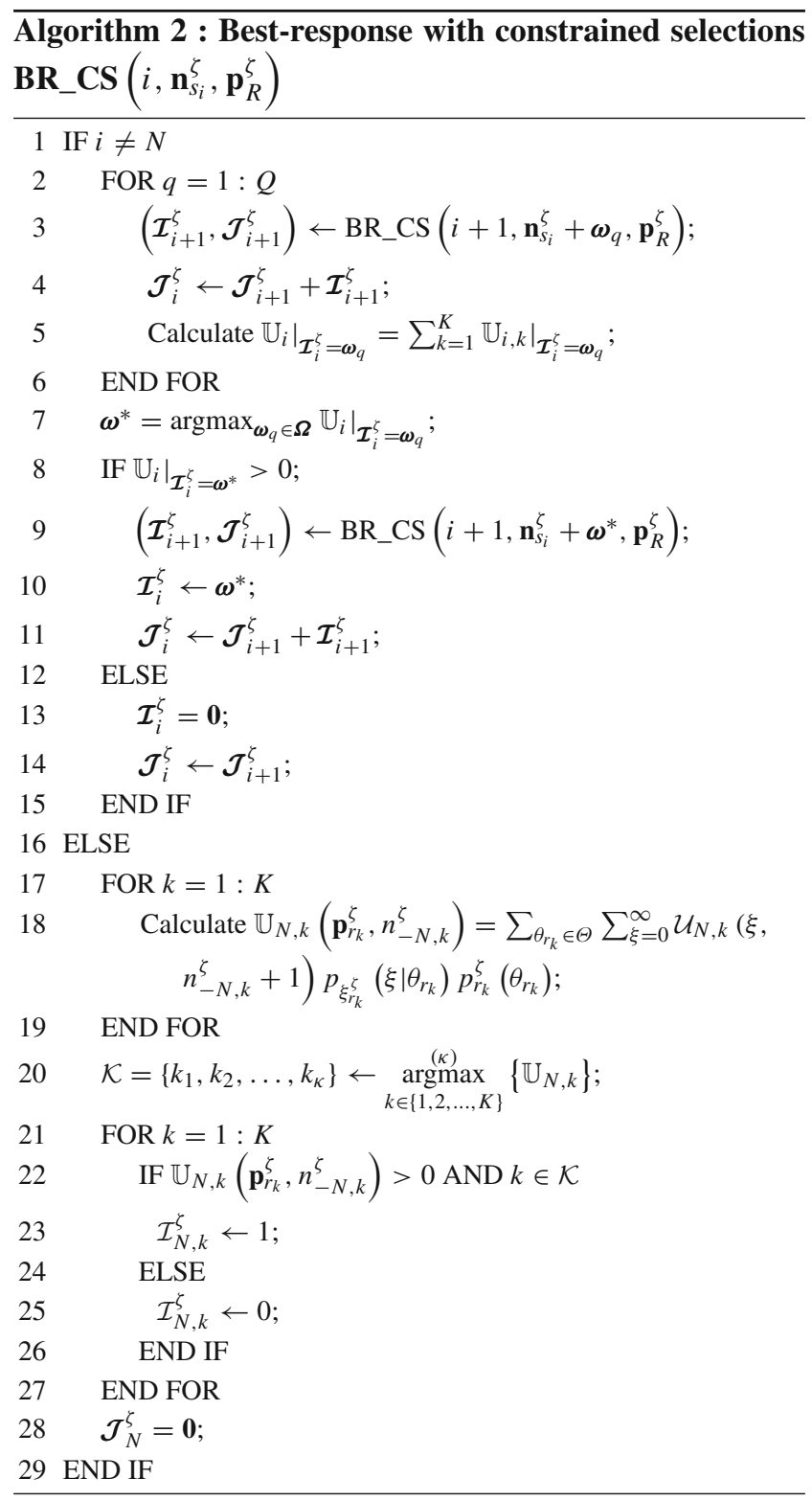




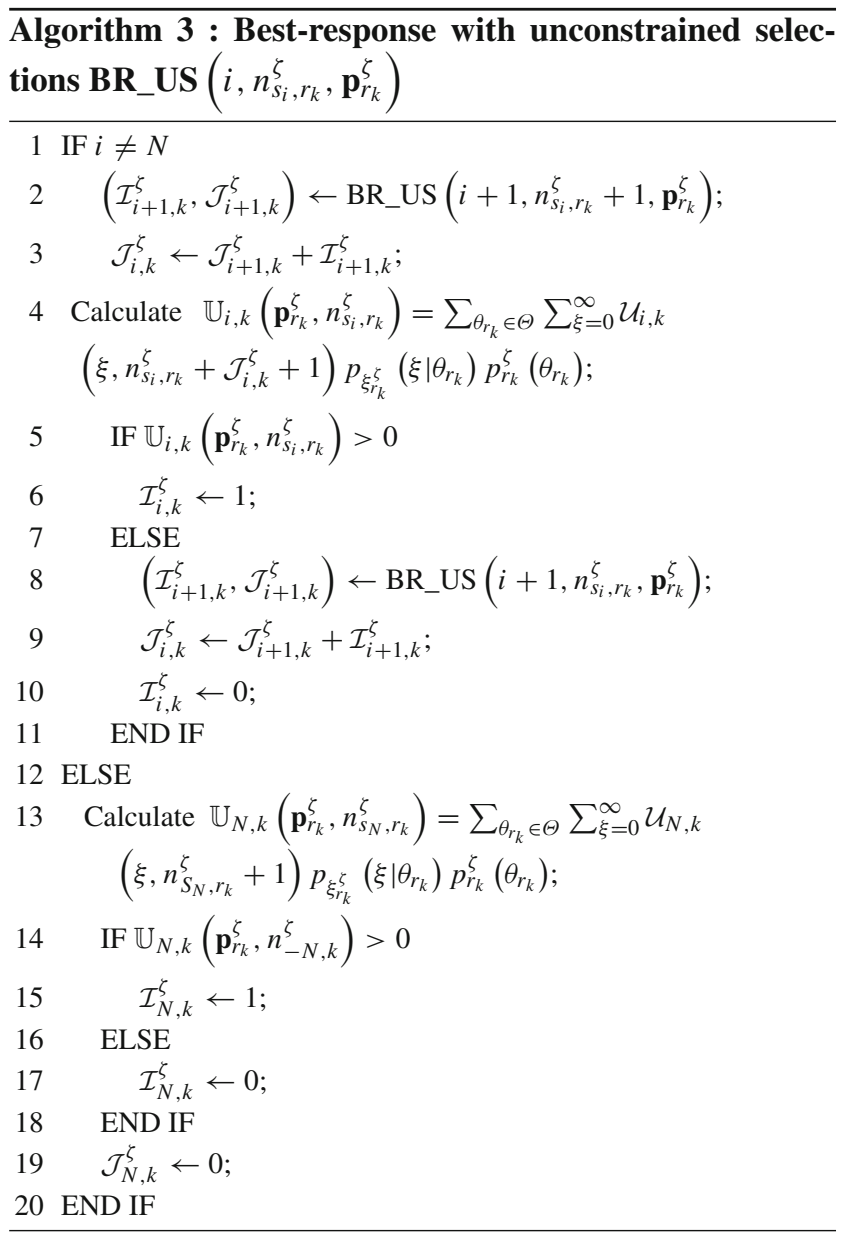

Publisher's Note Springer Nature remains neutral with regard to jurisdictional claims in published maps and institutional affiliations. 